\title{
A simple and rapid test of physical performance in chronic obstructive pulmonary disease
}

\author{
This article was published in the following Dove Press journal: \\ International journal of COPD \\ 2 August 2016 \\ Number of times this article has been viewed
}

\author{
Ali Mufraih Albarrati' \\ Nichola S Gale' \\ Stephanie Enright' \\ Margaret M Munnery ${ }^{2}$ \\ John R Cockcroft ${ }^{2}$ \\ Dennis J Shale ${ }^{2}$
}

'Physiotherapy Department, School of Healthcare Sciences, University Hospital of Wales, Cardiff University, Cardiff, UK; ${ }^{2}$ Cardiorespiratory Medicine Department, Cardio-Respiratory Medicine, Wales Heart Research Institute, Cardiff University, University Hospital of Wales, Cardiff, UK
Correspondence: Ali Mufraih Albarrati Department of Rehabilitation Sciences, College of Applied Medical Sciences, King Saud University, PO Box 10219 , Riyadh I 1433, Kingdom of Saudi Arabia Tel +966 I I469 6I28

Email abarrati@ksu.edu.sa

\begin{abstract}
Impaired physical performance is common in chronic obstructive pulmonary disease (COPD), but its assessment can be difficult in routine clinical practice. We compared the timed up and go (TUG) test and other easily applied assessments of physical performance with the 6-minute walk distance (6MWD). In a longitudinal study of comorbidities in COPD, submaximal physical performance was determined in 520 patients and 150 controls using the TUG test and 6MWD. Spirometry, body composition, handgrip strength, the COPD assessment test, St George's Respiratory Questionnaire (SGRQ), and the modified Medical Research Council dyspnoea scale were also determined. Patients and controls were similar in age, body mass index, and sex proportions. The TUG in the patients was greater than that in the control group, $P=0.001$, and was inversely related to $6 \mathrm{MWD}(r=-0.71, P<0.001)$ and forced expiratory volume in one second predicted $(r=-0.19, P<0.01)$ and was directly related to the SGRQ activity $(r=0.39, P<0.001)$, SGRQ total $(r=0.37, P<0.001)$, and total COPD assessment test scores $(r=0.37, P<0.001)$. The TUG identified the difference in physical performance between patients and controls. The TUG test and validated questionnaires provide a measure of physical performance, which is rapid and could be used in clinical practice.
\end{abstract}

Keywords: COPD, physical inactivity, timed up and go test

\section{Background}

A major impact of chronic obstructive airway disease is the progressive loss of physical performance, which may lead to disability with loss of the ability to perform routine activities of daily living. ${ }^{1,2}$ Reduced physical activity occurs even in mild severity airflow obstruction as demonstrated by continuous activity monitoring where patients spend less time walking and standing than healthy controls. ${ }^{3,4}$ In addition to the impact on functional status, physical inactivity is associated with a reduced health-related quality of life (HR-QoL) and contributes to loss of muscle mass, increased systemic inflammation, osteoporosis, and cardiovascular disease, all comorbidities of chronic obstructive pulmonary disease (COPD).${ }^{5,6}$ Consequently, it becomes a part of a vicious cycle of physical inactivity and changes in body composition that affect physical performance. ${ }^{5}$

The assessment of physical performance in patients with COPD in routine clinical practice is challenging and is often poorly quantified. Routine measurement of lung function, particularly the forced expiratory volume in 1 second $\left(\mathrm{FEV}_{1}\right)$, cannot predict the level of physical impairment. Physical inactivity has detrimental changes on body composition, including musculoskeletal wasting, which could have major impacts on HR-QoL. Earlier lifestyle interventions in the disease may maintain physical activity levels and a normal body composition. ${ }^{7}$ 
Physical performance in COPD has been assessed by various methods including questionnaires, which may have limited reproducibility and validity due to their dependence on patient recall. Quantitative measures of physical performance including 6-minute walking distance (6MWD), incremental shuttle walk test, and cycle ergometry are available but require substantial time, space, and expertise. ${ }^{8,9}$ The $6 \mathrm{MWD}$ is a validated measure of submaximal physical performance and reflects daily activities, but only commonly used in clinical research. ${ }^{10}$ The timed up and go (TUG) test is a relatively simple and reproducible test that assesses balance, gait speed, and physical performance and can predict of the risk of falls in COPD and elderly. ${ }^{11,12}$ However, the application of TUG in clinical practice is very limited, and only one study showed that TUG is feasible to be incorporated in the assessment of functions in patients with COPD. ${ }^{13}$ Thus, TUG may reflect routine daily activities, which require the integration of strength and balance.

We hypothesized that patients with COPD would have greater TUG than a non-COPD control population. Additionally, that, TUG would be related to $6 \mathrm{MWD}$ and other assessments of physical performance in a similar way to that reported in elderly individuals. The aim of this study was to examine the use of TUG test in COPD as a measure of physical performance and its association with 6MWD and other validated outcome measures in COPD.

\section{Methods}

\section{Subjects}

We assessed 520 patients with COPD, which was confirmed with spirometry and 150 controls, either current or ex-smokers free from cardiorespiratory and inflammatory diseases. ${ }^{14}$ This was a cross-sectional analysis from an ongoing longitudinal study of comorbidities and cardiovascular risk in COPD, Assessment of Risk in Chronic Airways Disease Evaluation (ARCADE, Clinical Trials No NCT 01656421). Patients were recruited when clinically stable and at least 4 weeks from an exacerbation of respiratory symptoms and free from other inflammatory diseases such as rheumatoid arthritis and inflammatory bowel disease. Controls were recruited from previous research databases carried out at the research centre and participant's relatives. The inclusion and exclusion criteria were previously published in the protocol paper of the ARCADE study. ${ }^{15}$ All the subjects gave written informed consent, and the study had approval from the South East Wales Research Ethics Committee.

\section{Anthropometry and body composition measurement}

All the subjects had their height measured barefoot using a stadiometer (Seca; Vogel \& Halke, Hamburg, Germany). Weight and body composition were recorded with subjects wearing lightweight clothing and barefoot using a single-frequency segmental bioelectrical impedance analyser (BC-418 MA; Tanita Corp., Tokyo, Japan). Body mass index (BMI, $\mathrm{kg} / \mathrm{m}^{2}$ ), fat-free mass (FFM), and fat mass (FM) were also determined. Waist and hip circumferences were measured with a stretch resistance tape. ${ }^{16}$

\section{Pulmonary function tests}

All the subjects completed spirometry and the $\mathrm{FEV}_{1}$, the forced vital capacity $(\mathrm{FVC})$, and $\mathrm{FEV}_{1} / \mathrm{FVC}$ ratio were recorded (Vitalograph alpha, Bucks, UK). Patients were asked to withhold their inhaler medication including bronchodilators for at least 6 hours prior to their visit but were given $400 \mu \mathrm{g}$ of salbutamol through a spacer device 10 minutes prior to the test.

In the patients, breathlessness was scored using the modified Medical Research Council (mMRC) dyspnoea scale and the number of exacerbations, defined as an acute worsening of respiratory symptoms characterized by the increase of any combination of three key symptoms that necessitate a change in regular medication, was recorded in the last year. ${ }^{17}$

\section{MWD}

The 6MWD was performed once in the subjects, and it was carried out in accordance with a protocol adapted from the American Thoracic Society guideline using a 30-m level, straight indoor track. ${ }^{18}$

\section{TUG}

All the subjects undertook the TUG test once (after demonstration) using a standard chair (height of the seat being $45 \mathrm{~cm}$ ) and standardized instructions. ${ }^{11}$ Subjects were seated with their back supported against the chair. They were instructed to stand up, walk $3 \mathrm{~m}$ to a mark on the floor, cross the mark, turn around, walk back to the chair, and sit down. The task had to be performed at their normal comfortable pace. A stopwatch was started on the word "go" and stopped as the subject sat down; the time was recorded in seconds.

\section{Hand grip measurement}

From a standing position with elbow extended, maximal right and left handgrip strength (HGS) was determined twice using a hand dynamometer, and the mean was calculated for 
each hand (Takei equipment industrial T.K.K.5401 grip-D; Takei, Japan).

\section{Health-related questionnaire}

Patients completed the St George's Respiratory Questionnaire (SGRQ) and the COPD assessment test (CAT), both are validated questionnaires to assess the impact of COPD on their health status. ${ }^{19,20}$

\section{Inflammatory biomarkers}

A blood sample was obtained for the determination of C-reactive protein (CRP, high sensitivity) and fibrinogen by standard assays (Department of Biochemistry, University Hospital of Wales).

\section{Statistical analysis}

The statistical software package SPSS 18.0 (SPSS Inc., Chicago, IL, USA) was used for all the analyses. Data were checked for normality prior to analysis. Parametric data were presented as mean and standard deviation or median (range) for nonparametric and categorical data. Comparisons between patients and controls were performed using analysis of variance. Categorical data were analyzed using the Chi-square test. Relationships between variables were explored using Pearson's $(r)$ and Spearman $\left(r_{s}\right)$ correlation coefficients. The correlation strength was classified as low $(0-0.25)$, moderate $(>0.25-0.50)$, strong $(>0.50-0.75)$, and very strong $(>0.75)$. Multivariate analysis was performed using a stepwise multiple regression model. For all the analysis, $P<0.05$ was considered significant. Receiver operating characteristics curve was performed to determine the diagnostic ability of the TUG test for the discrimination between stable patients with COPD and community population.

\section{Results}

The patients and controls were similar in age, sex ratio, and BMI. The patients had a greater tobacco exposure, lower mean $\mathrm{FEV}_{1}, \mathrm{FVC}$, and resting oxygen saturation than the control group, all $P<0.001$ (Table 1). The severity of airflow obstruction by Global initiative for chronic Obstructive Lung Disease (GOLD) stratification was GOLD $1 \mathrm{n}=70$, GOLD 2 $n=269$, GOLD $3 n=146$, and GOLD $4 n=35$. The patients were also subdivided according to GOLD quadrant based on the CAT score: GOLD A $=40$, GOLD B $=128$, GOLD C $=26$, and GOLD D $=326$.

\section{Measures of physical performance}

The patients had a greater TUG (mean \pm standard deviation: $11.5 \pm 4$ seconds) than the controls $(8.3 \pm 1.3$ seconds,
Table I Characteristics of patients with COPD and control subjects

\begin{tabular}{|c|c|c|c|}
\hline Variable & $\begin{array}{l}\text { COPD } \\
(n=520)\end{array}$ & $\begin{array}{l}\text { Control } \\
(n=150)\end{array}$ & $P$-value \\
\hline Sex (male:female) & $270: 250$ & $76: 74$ & 0.451 \\
\hline Age (years) & $66.1 \pm 7.6$ & $65 \pm 7.4$ & 0.109 \\
\hline $\mathrm{FEV}_{1} / \mathrm{FVC}(\mathrm{L})$ & $0.53 \pm 0.11$ & $0.78 \pm 0.05$ & $<0.001$ \\
\hline $\mathrm{FEV}_{1}$ (\% predicted) & $58 \pm 19$ & $105 \pm 14$ & $<0.001$ \\
\hline FVC (\% predicted) & $87 \pm 21$ & $109 \pm 15$ & $<0.001$ \\
\hline Smoking (pack-years) & $4 I \pm 25$ & $22 \pm 18$ & $<0.001$ \\
\hline BMI $\left(\mathrm{kg} / \mathrm{m}^{2}\right)$ & $28.0 \pm 5.5$ & $28.1 \pm 4.1$ & 0.951 \\
\hline Waist circumference $(\mathrm{cm})$ & $99.6 \pm 15.0$ & $94.7 \pm 10.2$ & 0.001 \\
\hline Hip circumference $(\mathrm{cm})$ & $104.2 \pm 11.0$ & $105.0 \pm 8.7$ & 0.250 \\
\hline Fat $\%$ & $34.1 \pm 8.4$ & $33.3 \pm 7.8$ & 0.345 \\
\hline FFMI $\left(\mathrm{kg} / \mathrm{m}^{2}\right)$ & $\mid 8.1 \pm 2.6$ & $18.5 \pm 2.3$ & 0.097 \\
\hline Handgrip (kg) & $27.1 \pm 9.7$ & $31.3 \pm 10.3$ & $<0.001$ \\
\hline TUG (seconds) & $11.5 \pm 4$ & $8.3 \pm 1.2$ & $<0.001$ \\
\hline 6MWD (m) & $335 \pm 125$ & $502 \pm 85$ & $<0.001$ \\
\hline Resting $\mathrm{O}_{2}$ saturation (\%) & $97 \pm 2$ & $98 \pm 1$ & $<0.001$ \\
\hline Fibrinogen $(\mathrm{g} / \mathrm{L})^{\#}$ & $3.5 \mathrm{I} \pm \mathrm{I} .3 \mathrm{I}$ & $3.08 \pm 1.25$ & $<0.001$ \\
\hline $\mathrm{CRP}(\mathrm{mg} / \mathrm{ml})^{\#}$ & $3.49 \pm 2.89$ & $1.76 \pm 3.18$ & $<0.001$ \\
\hline No exacerbations/year* & $2(I-3)$ & - & - \\
\hline SGRQ total* & $53(36-68)$ & - & - \\
\hline CAT score* & $2 \mid(14-27)$ & - & - \\
\hline
\end{tabular}

Notes: All data are mean (SD) unless otherwise indicated. *Represents median (range) and "represents geometric mean.

Abbreviations: 6MWD, 6-minute walk distance; BMI, body mass index; CAT, COPD assessment test; COPD, chronic obstructive pulmonary disease; CRP, C-reactive protein; FEV , forced expiratory volume in I second; FVC, forced vital capacity; FFMl, fat-free mass index; SD, standard deviation; SGRQ, St George's Respiratory Questionnaire; TUG, timed up and go; -, not applicable.

$P=0.001$ ), and 6MWD and HGS were less in patients than controls, both $P<0.001$ (Table 1). Across all the age decades $<49->70$ years, the patients had greater TUG than the controls, $P<0.001$ (Figure 1). Using the upper 95\% confidence interval (CI) for the control (8.42 seconds) as a cutoff value for the non-COPD range demonstrated that only 92 of the 520 patients had a TUG within the control reference range; however, there was no difference in the TUG for males and females in either group. Across the GOLD quadrant, the TUG test was greater in group D, $12 \pm 4.9$ seconds compared to group A, $8.9 \pm 2.4$ seconds, and group C, $9.4 \pm 2.3$ seconds, $P<0.05$, but was similar to group $\mathrm{B}, 11 \pm 4.4$ seconds. Using the cutoff value of 8.42 seconds, only $12 \%$ of patients in group D and $15 \%$ of group $\mathrm{B}$ were below the reference range. Although groups $\mathrm{A}$ and $\mathrm{C}$ had good lung function, $45 \%$ of group $\mathrm{A}$ and $69 \%$ of group C had greater TUG than the reference range.

The TUG was related to age in patients and controls (Table 2). In patients, the TUG was directly related to the $\mathrm{FEV}_{1} \%$ predicted, oxygen saturation, and modified Medical Research Council (mMRC) dyspnoea score and was inversely related to the 6MWD and HGS, whereas in the control group, 


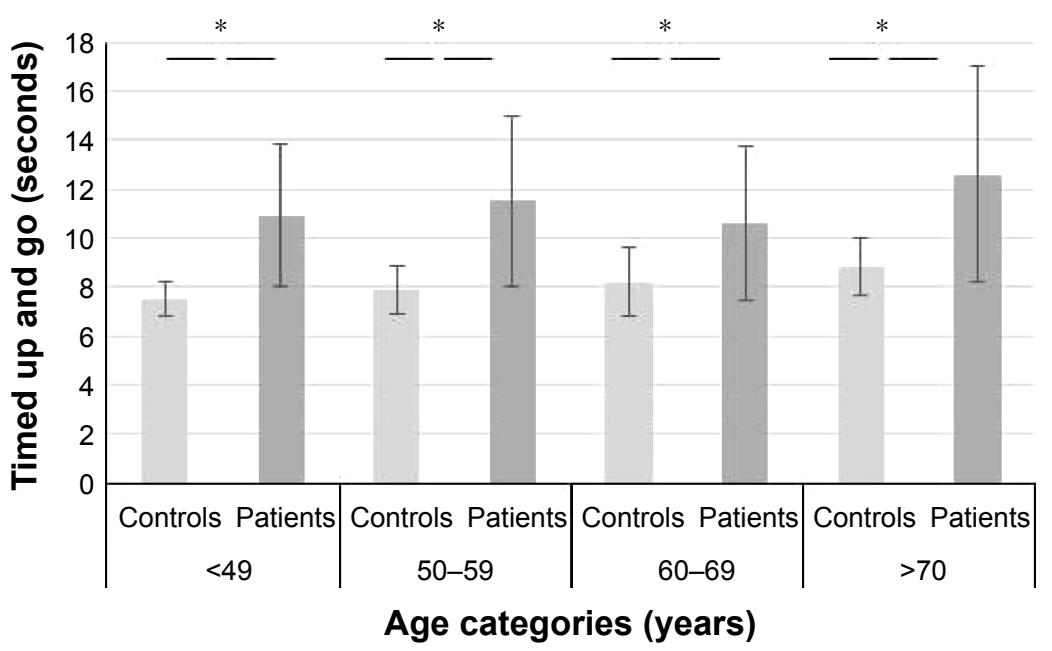

Figure I TUG test across age categories in COPD and control subjects.

Note: $* P<0.001$.

Abbreviations: COPD, chronic obstructive pulmonary disease; ROC, receiver operating characteristic; TUG, timed up and go.

it was inversely related only to the 6MWD (Table 2). The 6MWD, HGS, and TUG were all related to one another in the patients, $P<0.001$.

\section{Body composition and the TUG test}

In the patient group, the TUG was related to BMI, FM, FMI, and waist-to-hip ratio, whereas in the control group, it was only related to BMI and waist circumference (Table 2). Both TUG and 6MWD were related to FFM:FM ratio, $r=-0.13$, $P=0.01, r=0.11, P=0.04$, respectively, in the patient group, but only with 6MWD in the control group, $r=0.34, P=0.001$.

\section{Health-related questionnaires and TUG test}

The TUG and the SGRQ total score were moderately related $(r=0.37, P<0.001)$, as were the domains of activity ( $r=0.39, P<0.001)$, symptoms $(r=0.39, P<0.001)$, and

Table 2 Relationships between timed up and go test and other variables in patients and controls

\begin{tabular}{lllll}
\hline Variable & COPD & $P$-value & Control & $P$-value \\
\hline Age (years) & 0.18 & 0.005 & 0.22 & 0.001 \\
FEV \% predicted & -0.19 & 0.001 & -0.04 & 0.610 \\
Resting $\mathrm{O}_{2}$ saturation $(\%)$ & -0.12 & 0.005 & -0.002 & 0.977 \\
mMRC & 0.34 & 0.001 & - & - \\
6MWD (m) & -0.71 & 0.001 & 0.38 & 0.001 \\
HGS $(\mathrm{kg})$ & -0.27 & 0.001 & -0.02 & 0.833 \\
BMI $\left(\mathrm{kg} / \mathrm{m}^{2}\right)$ & 0.24 & 0.001 & 0.20 & 0.018 \\
FM $(\mathrm{kg})$ & 0.21 & 0.001 & 0.12 & 0.141 \\
FMI $\left(\mathrm{kg} / \mathrm{m}^{2}\right)$ & 0.21 & 0.001 & 0.12 & 0.144 \\
\hline
\end{tabular}

Abbreviations: 6MWD, 6-minute walk distance; BMl, body mass index; COPD, chronic obstructive pulmonary disease; HGS, handgrip strength; $\mathrm{FEV}_{l}$, forced expiratory volume in I second; FM, fat mass; FMI, fat mass index; mMRC, modified Medical Research Council; -, not applicable. impact $(r=0.32, P<0.001)$. The CAT score was also moderately related to the TUG $(r=0.37, P<0.001)$. Both the 6MWD and HGS were also related to the total SGRQ score, $r=-0.59$ and $r=-0.26$, respectively (both $P<0.001$ ) and similar to the CAT score $r=-0.53$ and $r=-0.27$, respectively (both $P<0.001$ ).

\section{Systemic inflammation}

Circulating CRP and fibrinogen were greater in patients than in controls $(P<0.001)$ and both were related to the TUG, CRP, $r_{\mathrm{s}}=0.19, P=0.001$, and fibrinogen, $r_{\mathrm{s}}=0.17, P<0.001$, but were unrelated to TUG in the control group. In the patients, 6MWD was also related to both CRP, $r_{\mathrm{s}}=0.21$, $P=0.001$, and fibrinogen, $r_{\mathrm{s}}=-0.27, P=0.001$, while neither biomarker was related to HGS.

\section{Frequency of exacerbation}

Of the patients, 210 reported zero to one exacerbation/year and 310 reported two or more exacerbations/year. The TUG was related to the frequency of exacerbations $r_{s}=0.24, P<0.001$. Frequent exacerbators, $11.5 \pm 3.5$ seconds, had greater TUG time than infrequent exacerbators, $10.6 \pm 3.9$ seconds, and both were greater than the comparator group TUG.

\section{Predictive factors for the 6MWD}

In the patients, stepwise multivariate regression analysis after controlling for age and BMI showed that TUG, mMRC, and CAT score explained $54 \%$ of the variability in $6 \mathrm{MWD}$ with $\mathrm{FEV}_{1} \%$ predicted excluded from the analysis, adjusted $R^{2}=0.54, P<0.001$. The TUG explained $45 \%$ of the reduction in 6MWD and mMRC, and total CAT score explained $29 \%$ and $18 \%$ of the variability, respectively. 


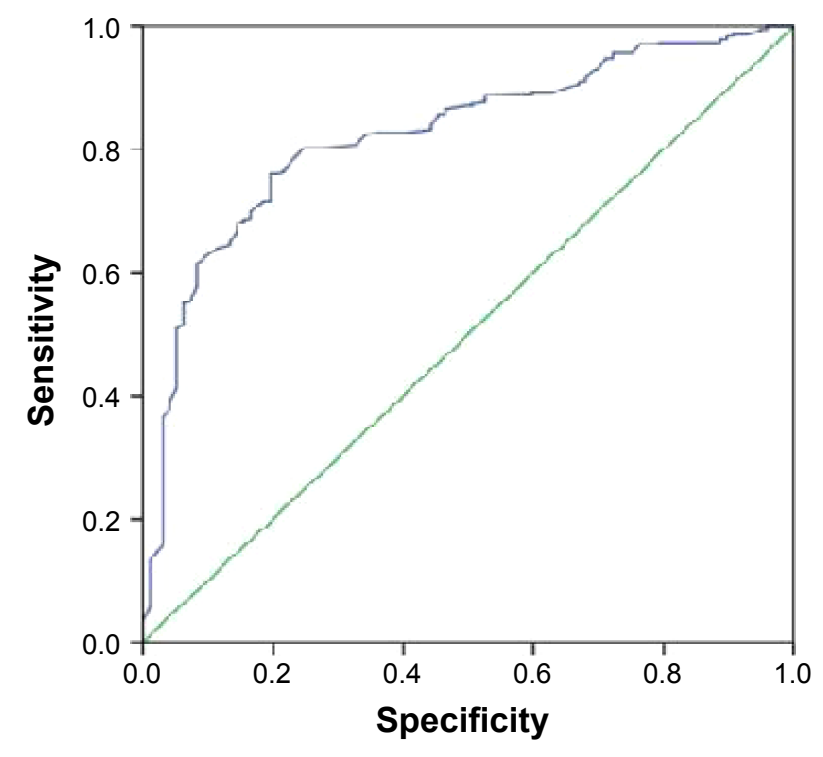

Figure 2 The ROC curve for the TUG test.

Note: Diagnostic ability of TUG test in patients with COPD.

Abbreviations: COPD, chronic obstructive pulmonary disease; ROC, receiver operating characteristic; TUG, timed up and go.

\section{Diagnostic ability of TUG test against 6MWD}

Using the upper 95\% CI (8.42 seconds) of the TUG for the control as a cutoff value for normal mobility, the TUG test showed an excellent diagnostic ability to predict the 6MWD as demonstrated by the area under the curve of 0.826 (0.783-0.870) (Figure 2). With 90\% sensitivity and $80 \%$ specificity, this cutoff value corresponds to $360 \mathrm{~m}$ on the 6MWD.

\section{Discussion}

The TUG test is an integrated assessment of physical function, which incorporates balance, gait speed, and functional capacity. ${ }^{11}$ In the present study, the TUG was greater in patients with COPD than a non-COPD control group and similar to age-stratified ranges reported in a meta-analysis of 21 studies in the elderly. ${ }^{21}$ By comparing the $95 \% \mathrm{CI}$ of both the groups, we suggest a cutoff point at 8.42 seconds or less for normal TUG test performance in this population. This threshold showed discriminative ability to identify patients with less physical performance with an area under the curve of 0.826 . Similarly, the TUG test was found to predict mobility status and reflect physical performance with an area under the curve of 0.969 in communitydwelling elderly women. ${ }^{22}$ Therefore, in clinical practice, patients who perform the TUG test in $>8.42$ seconds should receive a further evaluation of their physical status and early intervention to avoid subsequent complications related to physical inactivity.
A recent study showed that patients with COPD took longer time to perform the TUG than the controls, and a prolonged TUG identified patients with COPD at risk of falls. ${ }^{13}$ This is similar to the loss of physical performance, disability, and increased mortality risk shown in the elderly. ${ }^{23,24}$ Unlike other field tests of lower limb function in COPD such as sit to stand test and gait speed test, which only measure one dimension of lower limb function, the TUG test is an integrated measure, and in addition to lower limb function assessment, it measures balance and mobility. ${ }^{25,26}$ Its prolongation in COPD is a consequence of impairment of physical activity, skeletal muscle weakness, and deconditioning in a similar manner to that seen in the elderly. Thus in COPD, the TUG test is likely to reflect other measures of physical performance as well as the presence of comorbidities independent of the severity of airflow obstruction. ${ }^{4,13}$ This is consistent with previous studies, where the TUG test measured the interaction of body composition, muscle strength, and comorbidities on the physical performance. ${ }^{13,22}$ Hence, we explored the relationship of the TUG with the 6MWD, a measure of submaximal exercise capacity, and HGS as a surrogate marker for muscle strength. ${ }^{27}$ Both the measures were related to TUG, particularly the 6MWD, which had a strong relationship, indicating that the TUG in COPD similarly assesses impaired functional mobility. Other studies have found a strong relationship between the TUG and the 6MWD in patients with other chronic disease such as heart failure. ${ }^{13,28}$ The prolonged TUG in our patients may reflect lower limb muscle weakness, and this is supported by the lower HGS, a surrogate measure of peripheral muscle strength, which was associated with reduced physical activity in COPD. ${ }^{29}$ In healthy elderly populations where it has been associated with reduced lower extremity strength and less daily activity and predicts future disability. ${ }^{30}$ The relationship between the increased TUG and SGRQ and CAT scores indicates that the TUG reflects the impact of physical impairment and inactivity on patients' perceptions of their health and QoL. ${ }^{19,20}$

Altered body composition is an accepted comorbidity in COPD. Loss of skeletal muscle mass and function has been suggested as a cause of impaired physical function, similar to the sarcopenia and loss of physical function that occurs in healthy aging. ${ }^{31}$ Although FFM was not related to deficits in physical function in our study, changes in FM and its distribution were linked in both the controls and the patients. The TUG was related to abdominal obesity and FM, which is in line with studies showing that increased FM was the best predictor of functional limitation in COPD. ${ }^{32,33}$ This is supported by the UK study of the elderly similar to our cohort, 
which demonstrated that abdominal obesity determined as waist circumference was a major predictor of disability. ${ }^{34}$

The mechanisms linking FM and abdominal obesity to functional limitation may relate to the increased circulating levels of CRP and fibrinogen, which have been associated with reduced exercise capacity and left heart dysfunction in COPD. Circulating biomarkers, including CRP and fibrinogen, have been associated with physical decline and cardiovascular risk in older subjects. ${ }^{36}$ Fat produces various proinflammatory mediators including interleukin-6, a regulator of CRP production and secretion, and is overall likely to be a factor in the development of insulin resistance and the increased risk of diabetes mellitus and cardiovascular disease in COPD. ${ }^{5}$

The association of TUG and systemic inflammation in our patients suggests that the components of physical function measured by this test have a similar relationship to other assessments used in COPD. ${ }^{35}$ Systemic inflammatory biomarkers may also be a factor linking the TUG to the frequency of exacerbation when they are likely to increase.

The finding that the patients' TUG was independent of age and was greater than in the control group when stratified by decades, and also published age ranges could be interpreted as the loss of physical performance in COPD being further evidence of premature aging and is in keeping with evidence of premature vascular aging. ${ }^{37}$ Such an interpretation is further supported by the finding that over $80 \%$ of our patient group had a greater TUG than the upper $95 \% \mathrm{CI}$ of the control group. Confirming TUG as a valid indicator of disability in natural aging, a study in individuals over 85 years showed that having a TUG in the upper $10 \%$ percentile of a study population was associated with an increased risk of disability, odds ratio 9.02, and mortality. ${ }^{38}$ The predictive capacity of the TUG for similar outcomes in COPD will be an outcome of an ongoing prospective ARCADE study.

The assessment of physical function and consequent disability is clinically important in the management of COPD. However, most measures of physical performance are difficult to apply in clinical practice. They often require substantial space, equipment, and staff time, and some patients may not be able to maintain activity long enough to complete an assessment. The rapidity and simplicity of the TUG test suggests that it could be used to assess physical performance in the routine clinical settings. Furthermore, in elderly, the TUG test has been found to be a responsive measure to a rehabilitation exercise program. ${ }^{39,40}$ Combining the TUG test with an easily completed validated questionnaire, such as the CAT, as demonstrated here, could provide important information about a patient's physical performance and functional status that is not currently collected.

\section{Limitations}

A key limitation of this study is its cross-sectional nature, which limits understanding of the potential of this test in clinical practice. This issue is being addressed in a continuing longitudinal study (ARCADE). A limitation of measures of a number of physical functions is the requirement for time, space, and expertise. In addition, they may require substantial endurance, which limits the ability of patients to complete them. The short duration of TUG (ie, 1 minute including the instruction) removes the issues of endurance and resources that limit the application of some measures in COPD, and experience in the elderly suggests that it is a widely applicable assessment.

\section{Conclusion}

This large study confirmed that TUG, a simple valid measure of physical performance, was greater in COPD than controls. Implementing the TUG test and questionnaires in clinical practice may improve the overall management of COPD. We recommend a TUG cutoff of $>8.42$ seconds to highlight individuals requiring further evaluation and management of their physical status.

\section{Acknowledgments}

The authors would like to thank Dr James Duckers for his input. The study was funded by GlaxoSmithKline.

The current paper includes work based on the PhD thesis submitted by the main author Ali Mufraih Albarrati while at Cardiff University. The abstract of this paper was presented at the British Thoracic Society Conference as a poster presentation with interim findings. The poster's abstract was published in "Poster Abstracts" in Thorax Journal (http:// thorax.bmj.com/content/67/Suppl 2/A96.1).

\section{Disclosure}

The authors report no conflicts of interest in this work.

\section{References}

1. Maltais F, Decramer M, Casaburi R, et al. An Official American Thoracic Society/European Respiratory Society Statement: Update on limb muscle dysfunction in chronic obstructive pulmonary disease. Am J Respir Crit Care Med. 2014;189(9):e15-e62.

2. Eisner MD, Blanc PD, Yelin EH, et al. COPD as a systemic disease: impact on physical functional limitations. Am J Med. 2008;121(9): 789-796.

3. Gouzi F, Prefaut C, Abdellaoui A, et al. Evidence of an early physical activity reduction in chronic obstructive pulmonary disease patients. Arch Phys Med Rehabil. 2011;92(10):1611-1617.e2.

4. Watz H, Waschki B, Meyer T, Magnussen H. Physical activity in patients with COPD. Eur Respir J. 2009;33(2):262-272.

5. Barnes PJ, Celli BR. Systemic manifestations and comorbidities of COPD. Eur Respir J. 2009;33(5):1165-1185. 
6. Geffken DF, Cushman M, Burke GL, Polak JF, Sakkinen PA, Tracy RP. Association between physical activity and markers of inflammation in a healthy elderly population. Am J Epidemiol. 2001;153(3):242-250.

7. Pauwels RA, Buist AS, Calverley PM, Jenkins CR, Hurd SS. Global strategy for the diagnosis, management, and prevention of chronic obstructive pulmonary disease. NHLBI/WHO Global Initiative for Chronic Obstructive Lung Disease (GOLD) Workshop summary. Am J Respir Crit Care Med. 2001;163(5):1256-1276.

8. Palange P, Ward SA, Carlsen KH, et al. Recommendations on the use of exercise testing in clinical practice. Eur Respir J. 2007;29(1): 185-209.

9. Revill SM, Morgan MD, Singh SJ, Williams J, Hardman AE. The endurance shuttle walk: a new field test for the assessment of endurance capacity in chronic obstructive pulmonary disease. Thorax. 1999;54(3):213-222

10. Glaab T, Vogelmeier C, Buhl R. Outcome measures in chronic obstructive pulmonary disease (COPD): strengths and limitations. Respir Res. 2010;11:79.

11. Podsiadlo D, Richardson S. The timed "Up \& Go": a test of basic functional mobility for frail elderly persons. J Am Geriatr Soc. 1991;39(2): $142-148$.

12. Viccaro LJ, Perera S, Studenski SA. Is timed up and go better than gait speed in predicting health, function, and falls in older adults? $J \mathrm{Am}$ Geriatr Soc. 2011;59(5):887-892.

13. Al Haddad MA, John M, Hussain S, Bolton CE. Role of the timed up and go test in patients with chronic obstructive pulmonary disease. J Cardiopulm Rehabil Prev. 2016;36(1):49-55.

14. Global Initiative for Chronic Obstructive Lung Disease G. Global Strategy for the Diagnosis, Management and Prevention of COPD, Global Initiative for Chronic Obstructive Lung Disease (GOLD) [database on the Internet]; 2015. Available from: http://www.goldcopd.org. Accessed October 20, 2015.

15. Celli BR, MacNee W. Standards for the diagnosis and treatment of patients with COPD: a summary of the ATS/ERS position paper. Eur Respir J. 2004;23(6):932-946.

16. WHO. Waist circumference and waist-hip ratio: report of a who expert consultation; 2008 [cited 2012 01/12]. Available from: http://www.who.int/nutrition/publications/obesity/WHO_report_ waistcircumference_and_waisthip_ratio/en/index.html. Accessed April 16, 2014

17. Rabe KF, Hurd S, Anzueto A, et al. Global strategy for the diagnosis, management, and prevention of chronic obstructive pulmonary disease: GOLD executive summary. Am J Respir Crit Care Med. 2007;176(6):532-555.

18. ATS Committee on Proficiency Standards for Clinical Pulmonary Function Laboratories. ATS statement: guidelines for the six-minute walk test. Am J Respir Crit Care Med. 2002;166(1):111-117.

19. Jones PW, Harding G, Berry P, Wiklund I, Chen WH, Kline Leidy N. Development and first validation of the COPD Assessment Test. Eur Respir J. 2009;34(3):648-654.

20. Jones PW, Quirk FH, Baveystock CM, Littlejohns P. A self-complete measure of health status for chronic airflow limitation. The St. George's Respiratory Questionnaire. Am Rev Respir Dis. 1992;145(6):1321-1327.

21. Bohannon RW. Reference values for the timed up and go test: a descriptive meta-analysis. J Geriatr Phys Ther. 2006;29(2):64-68.

22. BischoffHA, Stahelin HB, Monsch AU, et al. Identifying a cut-off point for normal mobility: a comparison of the timed "up and go" test in communitydwelling and institutionalised elderly women. Age Ageing. 2003; 32(3):315-320.

International Journal of COPD

\section{Publish your work in this journal}

The International Journal of COPD is an international, peer-reviewed journal of therapeutics and pharmacology focusing on concise rapid reporting of clinical studies and reviews in COPD. Special focus is given to the pathophysiological processes underlying the disease, intervention programs, patient focused education, and self management protocols.
23. Beauchamp MK, Hill K, Goldstein RS, Janaudis-Ferreira T, Brooks D Impairments in balance discriminate fallers from non-fallers in COPD. Respir Med. 2009;103(12):1885-1891.

24. Idland G, Engedal K, Bergland A. Physical performance and 13.5-year mortality in elderly women. Scand J Public Health. 2013;41(4): 102-108.

25. Jones SE, Kon SSC, Canavan JL, et al. The five-repetition sit-to-stand test as a functional outcome measure in COPD. Thorax. 2013;68(11): 1015-1020.

26. Karpman C, Lebrasseur NK, Depew ZS, Novotny PJ, Benzo RP. Measuring gait speed in the out-patient clinic: methodology and feasibility. Respir Care. 2014;59(4):531-537.

27. Ling CH, Taekema D, de Craen AJ, Gussekloo J, Westendorp RG, Maier AB. Handgrip strength and mortality in the oldest old population: the Leiden 85-plus study. CMAJ. 2010;182(5):429-435.

28. Mesquita R, Janssen DJ, Wouters EF, Schols JM, Pitta F, Spruit MA. Within-day test-retest reliability of the timed up \& go test in patients with advanced chronic organ failure. Arch Phys Med Rehabil. 2013;94(11): 2131-2138.

29. Ansari K, Keaney N, Taylor I, Burns G, Farrow M. Muscle weakness, health status and frequency of exacerbations in chronic obstructive pulmonary disease. Postgrad Med J. 2012;88(1041):372-376.

30. Rittweger J, Beller G, Ehrig J, et al. Bone-muscle strength indices for the human lower leg. Bone. 2000;27(2):319-326.

31. Walston J, McBurnie MA, Newman A, et al. Frailty and activation of the inflammation and coagulation systems with and without clinical comorbidities: results from the Cardiovascular Health Study. Arch Intern Med. 2002;162(20):2333-2341.

32. Eisner MD, Blanc PD, Sidney S, et al. Body composition and functional limitation in COPD. Respir Res. 2007;8:7.

33. Kapella MC, Larson JL, Covey MK, Alex CG. Functional performance in chronic obstructive pulmonary disease declines with time. Med Sci Sports Exerc. 2011;43(2):218-224.

34. Angleman SB, Harris TB, Melzer D. The role of waist circumference in predicting disability in periretirement age adults. Int J Obes (Lond). 2006;30(2):364-373.

35. WatzH, WaschkiB, BoehmeC, Claussen M, MeyerT, Magnussen H. Extrapulmonary effects of chronic obstructive pulmonary disease on physical activity: a cross-sectional study. Am J Respir Crit Care Med. 2008;177(7) $743-751$.

36. Geffken DF, Cushman M, Burke GL, Polak JF, Sakkinen PA, Tracy RP. Association between physical activity and markers of inflammation in a healthy elderly population. Am J Epidemiol. 2001;153(3):242-250.

37. Sabit R, Bolton CE, Edwards PH, et al. Arterial stiffness and osteoporosis in chronic obstructive pulmonary disease. Am J Respir Crit Care Med. 2007;175(12):1259-1265.

38. Martin-Ruiz C, Jagger C, Kingston A, et al. Assessment of a large panel of candidate biomarkers of ageing in the Newcastle 85+ study. Mech Ageing Dev. 2011;132(10):496-502.

39. Lihavainen K, Sipila S, Rantanen T, et al. Effects of comprehensive geriatric intervention on physical performance among people aged 75 years and over. Aging Clin Exp Res. 2012;24(4):331-338.

40. Brooks D, Davis AM, Naglie G. Validity of 3 physical performance measures in inpatient geriatric rehabilitation. Arch Phys Med Rehabil. 2006;87(1):105-110.

This journal is indexed on PubMed Central, MedLine and CAS. The manuscript management system is completely online and includes a very quick and fair peer-review system, which is all easy to use. Visit http://www.dovepress.com/testimonials.php to read real quotes from published authors. 